\title{
Redescription de l'Heligmosome du Loir (Glis glis L.)
}

\author{
par Marie-Claude DURETTE-DESSET
}

[Laboratoire de Zoologie (Vers) associé au C.N.R.S. (Pr A.-G. Chabaud), Muséum National d'Histoire Naturelle, 57, rue Cuvier, F, 75 -Paris, 5', et Laboratoire d'Ecologie Médicale et de Pathologie parasitaire ( $\mathrm{P}^{r}$ J.-A. Rioux), Faculté de Médecine, rue Auguste-Broussonet, F, 34 - Montpellier]

\begin{abstract}
Résumé
On retrouve, dans toute l'Europe (Allemagne, Russie, Sardaigne, France), le même Héligmosome, chez le Loir Glis glis L. Ce n'est pas un Heligmosomum sensu stricto, mais nous préférons, à titre provisoire, conserver le binôme « Heligmosomum gracile (Leuckart, 1842)».
\end{abstract}

\section{Summary}

The same heligmosomid is found all over Europe (Germany, Russia, Sardinia, France) in the Rodent Glis glis L. It is not an Heligmosomum sensu stricto, but, transitory, we prefer keep the taxon « Heligmosomum gracile (Leuckart, 1842)》.

\section{Matériel.}

1) $\mathrm{Au}$ cours de recherches helminthologiques faites à la Boriz Nouvelle dans l'Hérault (France), en juillet 1968, nous avons piégé six Glis glis L., tous parasités en petit nombre (1), par des Nématodes héligmosomes situés soit dans le duodénum, soit dans l'intestin grêle.

2) Un deuxième lot provient d'un Glis glis de Sardaigne et a été envoyé par le Professeur Biocca en 1962. Il est constitué par six ỡ et un fragment postérieur de $q$

(1) La répartition des spécimens pour chaque Loir es: la suivan'e: tubes $353 \mathrm{M}: 2$; $359 \mathrm{M}$ : $3 \delta ; 362 \mathrm{M}: 5 \delta, 5 \% ; 367 \mathrm{M}: 2 \delta ; 369 \mathrm{M}: 3$ larves du $4^{e}$ stade larvaire ; $370 \mathrm{M}: 12 \delta^{+}, 8 q$. 
(tube 720 F). Tous ces spécimens sont déposés dans les collections du Muséum National d'Histoire Naturelle de Paris.

\section{Description des adultes.}

Nématodes de petite taille, rouge vif à l'état vivant, présentant un enroulement senestre le long de la ligne ventrale. Cet enroulement est lâche et le diamètre des spirès augmente d'avant en arrière. Chez le $\delta$, il y a deux à trois tours de spires, chez la $q$ trois à quatre.

Les Vers, observés in vivo dans le tube digestif ouvert, se trouvent au milieu des villosités, leur tête et leur queue profondément enfoncées dans la muqueuse, l'axe de la spire disposé parallèlement à la lumière intestinale.

ETUDES DES ARÊTES : Le corps est parcouru longitudinalement par 16 arrêtes cuticulaires dans les deux sexes. Celles-ci débutent pour la plupart derrière la vésicule céphalique (fig. $1, \mathrm{~A}, \mathrm{~B}, \mathrm{C}$ ) et disparaissent, chez le $\sigma^{*}$, suivant les spécimens, à des

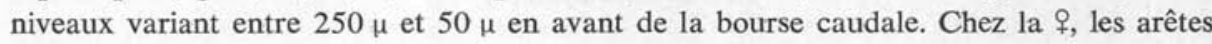
s'étendent jusqu'en avant de la vulve (fig. 1, D).

Ces arêtes se répartissent comme suit: une double arête gauche hypertrophiée, sept arêtes ventrales, sept arêtes dorsales (la plus proche du champ latéral gauche est également hypertrophiée), aucune arête en face du champ latéral droit. Il n'existe pas de gradient de taille des arêtes, mais elles ne sont pas non plus égales entre elles (fig. 1, F). La pointe des arêtes est dirigée de la droite vers la gauche pour les deux faces (fig. 1, F).

Dans la partie postérieure du corps et dans les deux sexes, la cuticule s'épaissit pour former des stries très marquées, transversales sur la face dorsale et longitudinales sur la face ventrale. Chez le $\hat{\delta}$, en coupe transversale, la pointe des arêtes ventrales est dirigée perpendiculairement à la paroi du corps, tandis que les arêtes dorsales gardent leur orientation droite-gauche (fig. 1, G). Chez la + , les arêtes, surtout les ventrales, s'épaississent et certaines s'anastomosent entre elles (fig. 1, D). En coupe transversale, la pointe des arêtes est dirigée perpendiculairement à la paroi du corps. (fig. 1, E).

Mâle: Corps long de $6,9 \mathrm{~mm}$, large de $200 \mu$ dans sa partie moyenne. Vésicule céphalique haute de $90 \mu$ sur $65 \mu$ de large. Anneau nerveux, pore excréteur et deirides situés respectivement à $240 \mu, 400 \mu$ et $340 \mu$ de l'apex. Esophage long de $580 \mu$ (fig. 1, A).

Spicules sub-égaux, longs de $2,2 \mathrm{~mm}$. Leurs extrémités distales, accolées chez certains spécimens, se présentent le plus souvent détachées l'une de l'autre et très souvent croisées comme l'indique la figure 2, D.

Suivant les spécimens, la bourse caudale peut avoir un aspect réticulé ou simplement présenter une trame très fine, visible à un fort grossissement. Cependant la surface cuticulaire est lisse. Nous pensons que le reticulum est dû à la coagulation du liquide protéique compris entre les deux feuillets de la bourse, coagulation dont l'importance varie suivant l'état de l'animal et surtout vraisemblablement la température à laquelle est faite la fixation. 

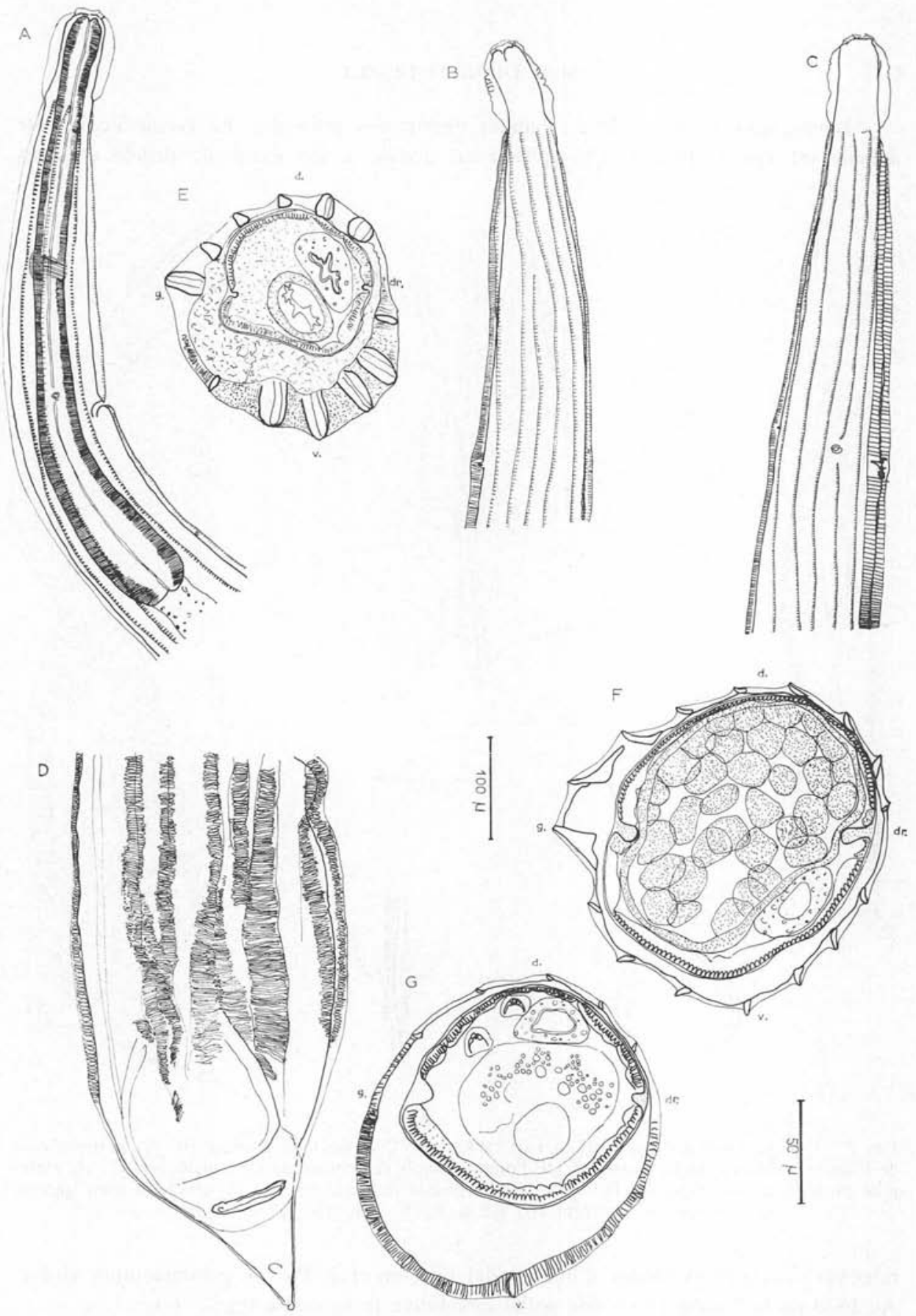

Fig. 1. - Heligmosomum gracile (Leuckart, 1842). A : $\delta$, extrémité antérieure, vue latérale droite.

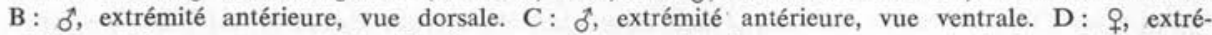
mité postérieure, vue ventrale. E: $\subsetneq$, coupe transversale du corps en avant de la vulve. F: $ᄋ$, coupe transversale au milieu du corps. G : $\delta$, coupe transversale dans la partie postérieure du corps. A, B, C, D : éch. $100 \mu ;$ E, F, G : éch. $50 \mu$ 
Bourse caudale symétrique. Papilles prébursales présentes. La racine de la côte dorsale est épaisse (fig. 2, C). Celle-ci est divisée à son extrémité distale en deux
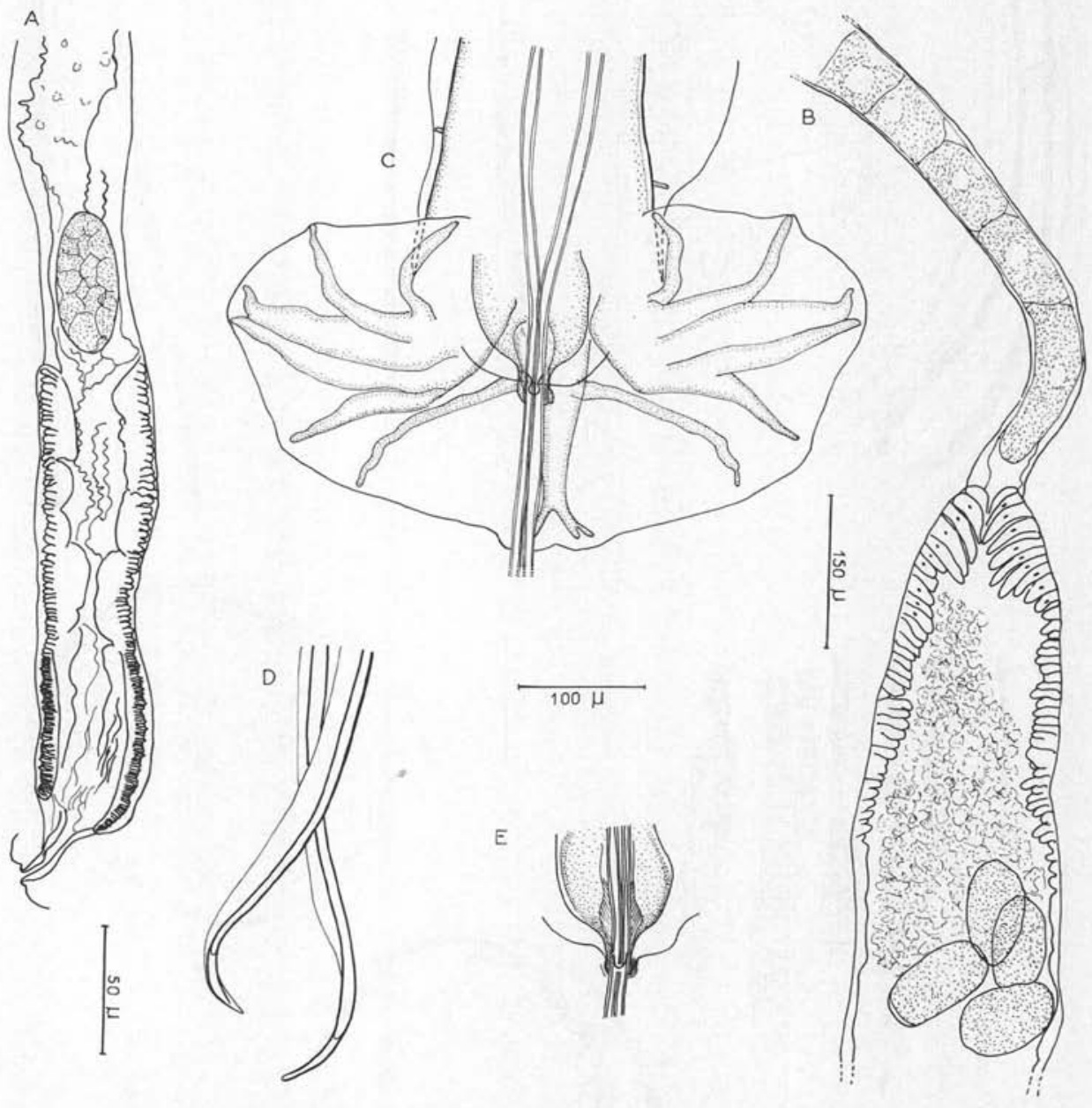

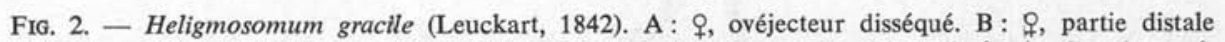
de l'ovaire, oviducte, partie proximale de l'utérus rempli de spermatozoïdes, disséqués. C: $\delta$, extré-

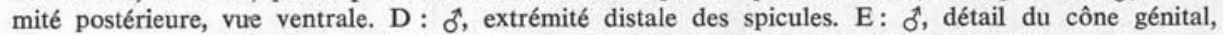
vue ventrale. - A: éch. $100 \mu$; B, C, E: éch. $150 \mu$; D: éch. $50 \mu$

rameaux, eux-mêmes bifides. Cône génital bien marqué. Pas de gubernaculum visible. $\mathrm{Au}$ fond de la bourse existe une petite membrane transversale (fig. 2, C).

Femelle: Corps long de 10,9 mm, large de $220 \mu$. Vésicule céphalique légèrement asymétrique, haute de $92 \mu$ (face ventrale), $95 \mu$ (face dorsale) sur $62 \mu$ de large. 
Anneau nerveux, pore excréteur et deirides situés respectivement à $250 \mu, 430 \mu$ et $425 \mu$ de l'apex. Esophage long de $560 \mu$.

Appareil génital monodelphe. La vulve s'ouvre à $110 \mu$ de la queue. L'ovéjecteur est d'un type très particulier car nous n'avons pu avec certitude en séparer les différentes parties, après dissection. Nous donnons donc les mesures suivantes sous réserve : vestibule : $155 \mu$, sphincter : $215 \mu$, trompe : $175 \mu$ (fig. 2 , A), utérus : $4 \mathrm{~mm}$. La portion proximale de l'utérus est nettement différenciée alors que l'oviducte est pratiquement inexistant (fig. 2, B).

Eufs allongés, au stade morula, longs de $110 \mu$ sur $50 \mu$ de large, et assez nombreux (une cinquantaine environ).

Queue courte, arrondie à l'extrémité, longue de $45 \mu$.

\section{Description des larves du quatrième stade :}

ETUDE DES ARÊTES: Le corps est parcouru longitudinalement par cinq arêtes cuticulaires qui débutent environ à $50 \mu$ en arrière de la vésicule céphalique (fig. $3, \mathrm{~A}$ ) et s'étendent jusqu'à $150 \mu$ chez le $\sigma$ (fig. 3 , E) et $250 \mu$ chez la $q$ (fig. 3 , D), en avant de la queue. Ces arêtes se répartissent comme suit: une arête gauche et une arête aroite, trois arêtes ventrales, la face dorsale est dépourvue d'arêtes (fig. 3, C). Ces arêtes sont sensiblement toutes de même taille. En coupe transversale, au milieu du corps, la pointe des arêtes est dirigée de la droite vers la gauche pour les deux faces (fig. 3, C).

Mâle : Longueur : 1,8 mm, largeur $90 \mu$; Anneau nerveux, pore excréteur et deirides situés respectivement à $150 \mu, 230 \mu$, et $230 \mu$ de l'apex. Esophage long de $280 \mu$.

Femelle : Longueur : $2,17 \mathrm{~mm}$, largeur : $50 \mu$; Anneau nerveux, pore excréteur et deirides situés respectivement à $190 \mu, 270 \mu$ et $270 \mu$ de l'apex. Esophage long de $365 \mu$. Queue longue de $82 \mu$.

\section{Discussion.}

Ainsi que l'indique Dujardin, 1845, \& Rudolphi inscrit au nombre de ses espèces douteuses un Strongylus myoxi (Synopis, p. 36, $\mathrm{n}^{\circ} 27$ ) qui aurait été porté sur un ancien catalogue du musée de Vienne et que nous ne trouvons pas imprimé en 1821 ». Par ailleurs, la mention de Rudolphi (1819) ne s'accompagne d'aucune description. Ce binôme est donc un nomen nudum.

En 1842, Friedrich S. Leuckart décrit un Strongle chez un Loir, originaire d'Allemagne et le nomme Strongylus gracilis. Sa description, qui est accompagnée de trois dessins, précise la taille des Vers : $6,75 \mathrm{~mm}$ pour le $\delta, 9 \mathrm{~mm}$ pour la ?. Leuckart remarque également l'épaississement de la partie postérieure du corps chez la $q$ et décrit les spicules comme deux longs fils. En outre, on peut observer sur son dessin que la côte dorsale est longue. 

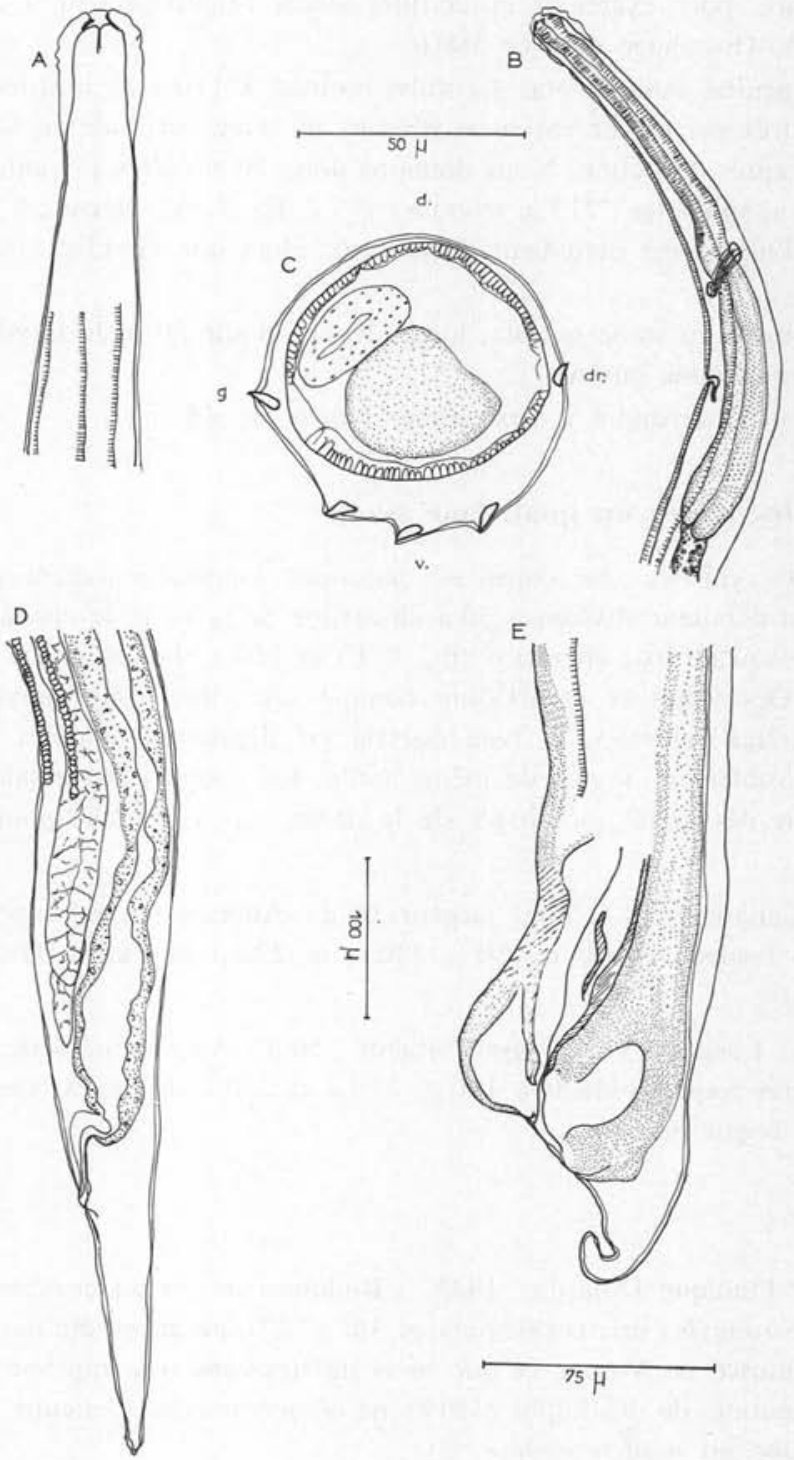

Fig. 3. - Heligmosomum gracile (Leuckart, 1842). Larves du $4^{\circ}$ stade. A : $q$, extrémité antérieure, vue ventrale, montrant le départ des arêtes cuticulaires. B : $\$$, extrémité antérieure, vue latérale gauche. $\mathrm{C}$ : $ᄋ$, coupe transversale au milieu du corps. D : $ᄋ$, extrémité postérieure, vue latérale gauche. E: $\delta$, extrémité postérieure, vue latérale gauche. - A, D, E: éch. $75 \mu$; B : éch. $100 \mu ; \mathrm{C}$ : éch. $50 \mu$ 
Dujardin en 1845, dans ses Suites à Buffon, signale l'espèce de Leuckart, mais pense qu'elle est identique à une des espèces qu'il a déterminées précédemment chez les Mulots et les Campagnols, sans cependant préciser laquelle.

Cette espèce, rangée d'abord dans le genre Metastrongylus par Molin en 1861 et considérée comme différente de Strongylus myoxi par Diesing, 1851 et Stossich, 1899, est finalement classée dans le genre Heligmosomum par Railliet et Henry, 1909. C'est dans ce genre qu'elle se trouve encore actuellement (cf. Skrjabin, Schikhobalova et Schulz, 1953).

Parmi les auteurs modernes, Hall en 1916 considère qu'Heligmosomum gracile est une espèce valide, ce que confirme Travassos en 1921. Pour ce dernier, l'inclusion dans le genre Heligmosomum d'H. gracile ne peut être que provisoire puisque la description de Leuckart ne précise pas si l'utérus est double ou non.

Deux autres espèces sont décrites actuellement chez le Loir, toutes les deux en Russie. Ce sont Longistriata schulzi, Schachnasarova, 1949, et Longistriata elpatievskii Schachnasarova, 1949. Nous pensons qu'en fait, ces deux espèces sont synonymes et identifiables à notre matériel. En effet, l'auteur avait séparé ses deux espèces parce que chez $L$. schulzi, la bourse caudale du ơ est réticulée alors qu'elle ne l'est pas chez L. elpatievskii. En fait, comme nous l'avons dit plus haut, la formation du réticule est due à un artefact de fixation. Les autres caractères correspondent avec notre matériel et l'assimilation de nos spécimens avec les espèces de Schachnasarova n'offre pas de difficultés.

L'identification de nos spécimens avec $H$. gracile s'impose également. En effet :

1) La description de Leuckart ne présente aucun point qui soit contraire à ce que nous avons observé chez nos spécimens. De plus, les mensurations du $\sigma^{\star}$ et de la $q$ concordent, les spicules sont décrits comme longs et fins et on peut voir sur un des dessins de Leukart que la dorsale est longue.

2) Nous avons vu plus haut que la répartition de l'Héligmosome du Loir est européenne puisqu'il a été trouvé en Russie, en Sardaigne, et en France. Il s'agit donc, selon toute probabilité ,de la même espèce en Allemagne et en Autriche.

\section{Conclusion.}

L'Héligmosome du Loir est donc une espèce valide qui se nomme actuellement Heligmosomum gracile (Leuckart, 1842), Railliet et Henry, 1909 et se trouve dans toute l'Europe. En fait, cette espèce n'est pas un Heligmosomum selon la définition du genre que nous avons donnée en 1968. En effet, elle possède une côte dorsale longue et elle ne présente pas le système des arêtes cuticulaires du genre Heligmosomum.

Nous serons amenée à la ranger, prochainement dans un genre particulier, mais nous préférons, avant d'effectuer cette opération, avoir une vue générale sur les Helig. mosomatidae du monde entier. 


\section{Bibliographie}

Diesing (K. M.), 1851. - Systema Helminthum, t. 2, Vindobonae, vI + 588 pp.

DUjardin (F.), 1845. - Histoire Naturelle des Helminthes ou vers intestinaux, Paris, XVI + 654 pp. +15 pl.

Durette-Desset (M.-C.), 1968. - Les systèmes d'arêtes cuticulaires chez les Nématodes héligmosomes. III. Etude de sept espèces parasites de Rongeurs néarctiques et rétablissement du genre Heligmosomoides Hall, 1916. Bull. Mus. Nat. Hist. Nat., 40, 186-209.

Hall (M. C.), 1916. - Nematode parasites of Mammals of the orders Rodentia, Lagomorpha and Hyracoidea. Proc. U.S. Nat. Mus. Wash., 50, 1-258.

LeUCKART (F.S.), 1842. - Helminthologische Beiträge (Zoologische Bruchstücke). Freiburg, $\mathrm{III}+60 \mathrm{pp}$.

Molin (R.), 1861. - Il Sottordine degli acrofalli ordinato scientificamente secondo i risultamenti delle indagini anatomiche ed embriogeniche. Mem. r. Hist. Veneto, di. Sci. lett. ed. arti. Venzia, 9, 427-633.

Railliet (A.) et Henry (A.), 1909. - Sur la classification des Strongylidae et Metastrongylidae. C.R. Soc. Biol., 66, 85-88.

RUDOLPHI (C. A.), 1819. - Entozoorum synopsis cui accedunt mantissa duplex et indices locupletissimi, Berolini, $\mathrm{x}+811 \mathrm{pp}$.

Schachnasarova (S. S.), 1949. - Novyje nematody gryzumov Azerbejdzana. Trudy gelm. labor., 2, 69-86.

SkrJabin (K. I.), Schikhobalova (N. P.) et Schulz (R. S.), 1953. - Osnovi nematodologii, 4, Acad. Nauk. SSSR, Moscou, 1954, 323 pp.

Stossich (M.), 1899. - Strongylidae. Boll. Soc. Adriatica Sc. Nat. Trieste, 55-152.

Travassos (L.), 1921. - Contribuiçoes para o conhecimenta da fauna helmintolojica brasileira. XIII. Ensaio monografico da familia Trichostrongylidae Leiper, 1909. Mem. Inst. Oswaldo Cruz, 13, 1-135. 